\title{
Operation and Performance of the ATLAS Silicon Microstrip Tracker
}

\section{Petra HAEFNER ${ }^{* \dagger}$}

Max-Planck-Institut für Physik

E-mail: Petra.Haefner@cern.ch

\begin{abstract}
The SemiConductor Tracker (SCT) is a precision tracking device in the ATLAS experiment made up from silicon microstrip sensors processed in planar p-in-n technology. The signal from the strips is processed in the front-end ASICs working in binary readout mode. Data is transferred to the off-detector readout electronics via optical fibres. It was installed in 2008 and has been operated under various conditions like cosmic ray, single proton, pp and heavy ion collision runs. Calibration data has been taken and analysed to determine the performance of the system. After the commissioning phase it arrived to the first LHC pp collision runs in very good shape: $99 \%$ of the SCT strips are operational, noise occupancy and hit efficiency exceed the design specifications and it is well timed-in. It is stably operating since the start of the physics program with a data taking efficiency of about $99.4 \%$ in $7 \mathrm{TeV}$ pp collisions. The detector geometry is monitored continuously and is extremely stable during standard operating conditions. In non-standard conditions movements of up to $5 \mu \mathrm{m}$ have been observed. After 1.5 years of running, first effects of irradiation have been measured in an increased leakage current. The measurement agrees well with predictions within the safety factor of $50 \%$ assumed for the upgrade development.
\end{abstract}

The 20th Anniversary International Workshop on Vertex Detectors

June 19-24, 2011

Rust, Lake Neusiedl, Austria

\footnotetext{
* Speaker.

$\dagger$ On behalf of the ATLAS Collaboration.
} 


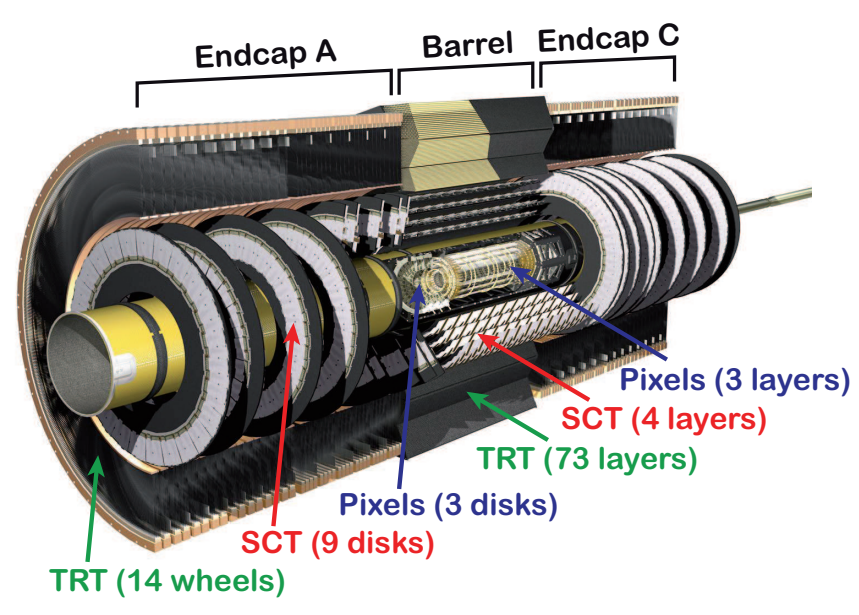

Figure 1: The Inner Detector of the ATLAS experiment [1] consisting of the pixel vertex detector, the silicon microstrip tracker (SCT) and the transition radiation tracker (TRT).

\section{Detector Setup}

The SemiConductor Tracker (SCT) is composed of three parts, the barrel and two endcaps (cf. Fig. 1). The barrel consists of four concentric cylinder layers equipped with 2112 silicon modules [2]. It covers the central pseudorapitity range up to $|\eta|=1.4$. The two endcaps consist of 9 disks each, equipped with 1976 modules in total [3]. The endcaps cover the forward pseudorapidity range up to $|\eta|=2.5$. The $\mathrm{SCT}$ detector is $5.4 \mathrm{~m}$ long and covers the radial range of $(30-52) \mathrm{cm}$. The full detector contains $61 \mathrm{~m}^{2}$ of active material and has around 6.3 million readout channels.

The modules are built from two single-sided sensors of $285 \mu \mathrm{m}$ thickness, glued back-toback at a stereo angle of $40 \mathrm{mrad}$ to allow the measurement of two-dimensional hit positions (cf. Fig. 2). $83 \%$ of the sensors were produced by Hamamatsu, whereas $17 \%$ are from CiS. Each sensor consists of two wafers bonded together and has 768 p-type strips on n-type silicon with a mean pitch of $80 \mu \mathrm{m}$ in the barrel and 57-94 $\mu \mathrm{m}$ in the endcaps. The barrel modules are rectangular shaped, whereas the endcaps sensors are trapezoidal. To allow for the different radii of the endcap disks, five types of endcap sensors were produced. The strip length is about $13 \mathrm{~cm}$ for the barrel modules and between (6-12) $\mathrm{cm}$ in the endcap ones. The SCT detector is designed to reach a $17 \mu \mathrm{m}$ hit resolution in the $r-\phi$ direction (bending plane) and a $580 \mu \mathrm{m}$ hit resolution in the z-direction along the beam pipe.

The sensors are mounted onto a baseboard out of $\mathrm{TPG}^{1}$ that serves as thermal conductor to remove the heat generated by readout electronics and sensors. The readout of the modules is performed by six radiation-hard front-end readout chips (ABCD3TA) per side [4]. Thus, each chip performs the readout of 128 strips. 48 modules are served by one ROD ${ }^{2}$. Eleven or twelve RODs send their data to one ATLAS ROS ${ }^{3}$. A so-called TIM ${ }^{4}$ provides trigger and clock to the RODs.

\footnotetext{
${ }^{1}$ Thermal Pyrolitic Graphite

${ }^{2}$ ReadOut Driver

${ }^{3}$ ReadOut Subsystem

${ }^{4} \mathrm{~T}$ (rigger)T(iming)C(ontrol) Interface Module
} 


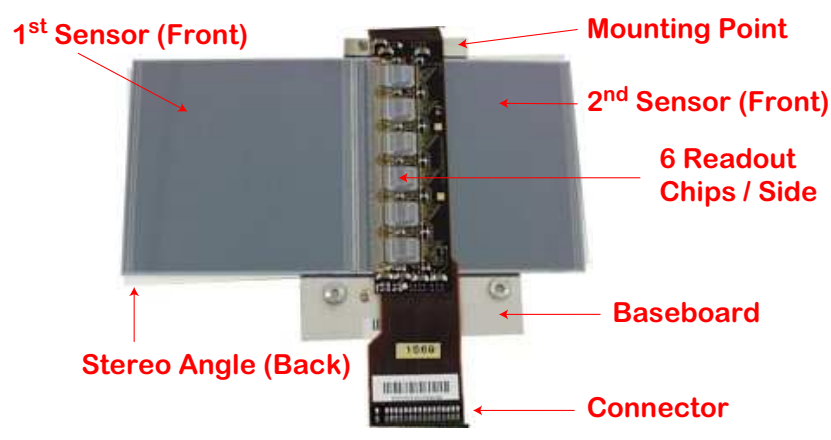

Figure 2: A barrel module of the SCT with its major components.

During stable proton collisions, the SCT detector is operated at a reverse bias voltage of $150 \mathrm{~V}$. At the beginning of operation, full depletion is reached at about $60 \mathrm{~V}$. This will increase with irradiation over the coming years (see Sect. 8). The maximal applicable voltage to the SCT modules is $500 \mathrm{~V}$. When LHC beams are non-stable the bias voltage is reduced to $50 \mathrm{~V}$ (standby) to minimize the risk of damaging the silicon modules by possible large charge deposits into the active material and the readout chips.

The silicon modules are cooled by an evaporative cooling system based on $C_{3} F_{8}$ [5]. The modules are operated at different temperatures depending on their position in the detector. The inner three barrel layers are cooled to $-1.5^{\circ} \mathrm{C}$. The outermost barrel layer is kept at a temperature of $4.5^{\circ} \mathrm{C}$ in order to act as a thermal shield to the TRT detector, that is operated at room temperature. The endcaps are kept at $-7^{\circ} \mathrm{C}$.

\section{Timing and Readout Modes}

The SCT modules are read out in binary mode, which registers whether or not the signal is above a programmable threshold of $1 \mathrm{fC}$. The SCT data acquisition reads out three time bins, where the trigger accept signal lies in the middle bin. A time bin is $25 \mathrm{~ns}$ long corresponding to the nominal LHC bunch spacing. The SCT readout can operate in three different timing modes. The first one "XXX" has no hit requirement and was used throughout 2010, when the minimum bunch distance was 75 ns. In 2011, since LHC operates with 50 ns bunch trains, the so-called "X1X" timing mode is applied. Here, a hit in the middle (i.e. trigger) time bin is required. For a well timed-in detector we expect no signal above threshold in the first bin (0), a signal above threshold in the second bin (1) and there is no requirement on the third bin (X). The third timing mode is therefore called "01X". This will be the standard SCT readout mode for the LHC design of $25 \mathrm{~ns}$ bunch trains. Those are currently planned from the 2012 run on.

In order to ensure that hits are arriving in the correct time bin, timing scans are performed for all modules. According to those, adjustments are made in the readout to compensate for differences in the optical fibre lengths and the time-of-flight from the interaction point to the individual module. The time delay for each module can be adapted in the TIM. A full scan comprises time offsets between $-20 \mathrm{~ns}$ to $+20 \mathrm{~ns}$ in $5 \mathrm{~ns}$ steps. Finer granularity $(1 \mathrm{~ns})$ can be achieved as well. An 


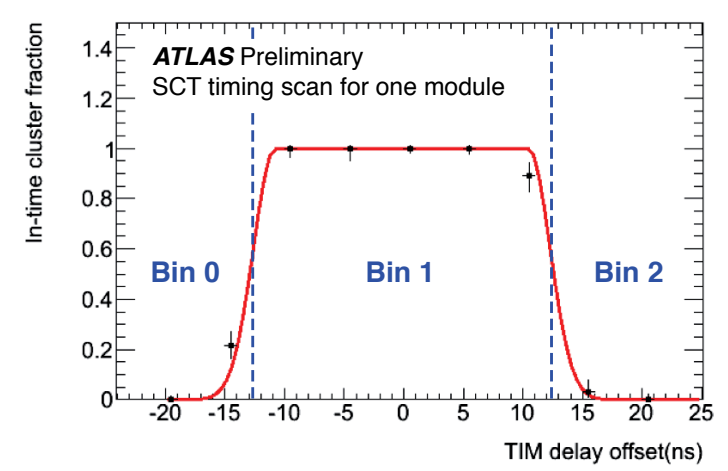

Figure 3: Timing scan for one SCT module. A well timed-in module has the middle of the plateau at zero.

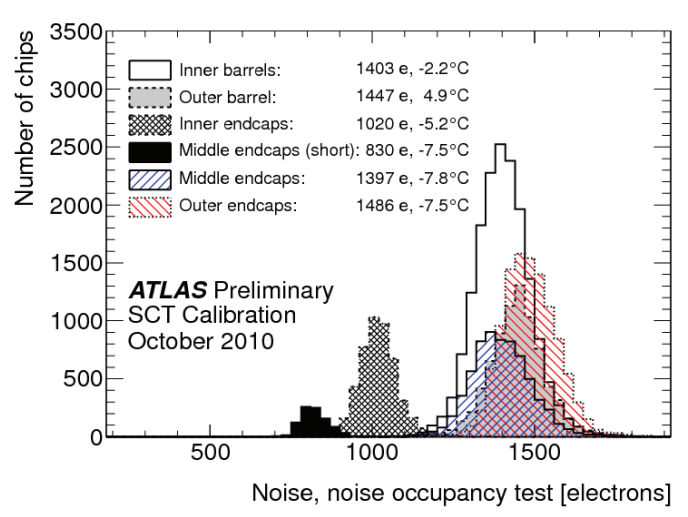

Figure 4: Distribution of noise measured per chip in the different parts of the SCT. Results are extracted from a response curve test.

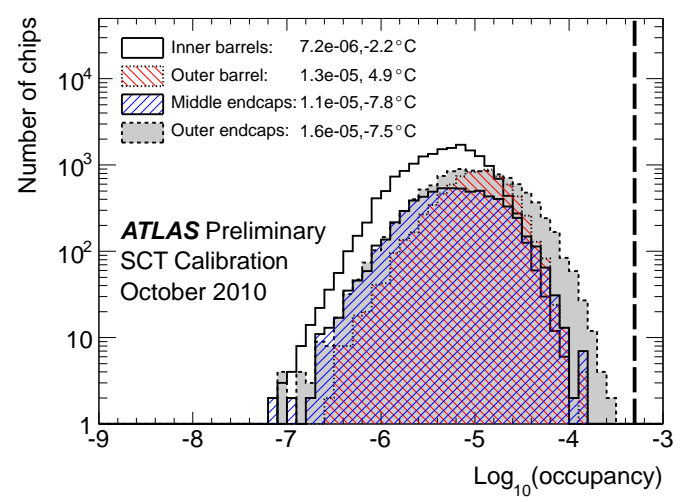

Figure 5: Noise occupancies per chip in the different parts of the SCT. The results are extracted from a noise occupancy test.

example of a scan for one module can be seen in Fig. 3. Timing scans for all modules have shown that the SCT is very well timed-in.

\section{Noise Occupancy}

The noise occupancy of the SCT detector is measured with two methods either with special calibration runs or during normal data taking using random triggers. For calibration, the SCT readout chips are equipped with a charge injection circuit that can apply charges of 0-16 fC. For a given injected charge, the occupancy vs. threshold is measured. The resulting S-curve is parametrized by a complementary error function. The width of that distribution characterizes the noise. Figure 4 shows an example of such a calibration run. Average values for each detector region are given together with the estimated average silicon temperature during the measurement. One can see that a typical noise level is around 1500 electrons, whereas the signal threshold corresponds to about 6200 electrons ( $1 \mathrm{fC}$ ). Consequently, the noise occupancy of the SCT detector is very low as shown in Fig. 5 using the second method. The noise occupancy is defined as the number of hits observed in random events divided by the number of (active) readout channels. Typical values are on the 


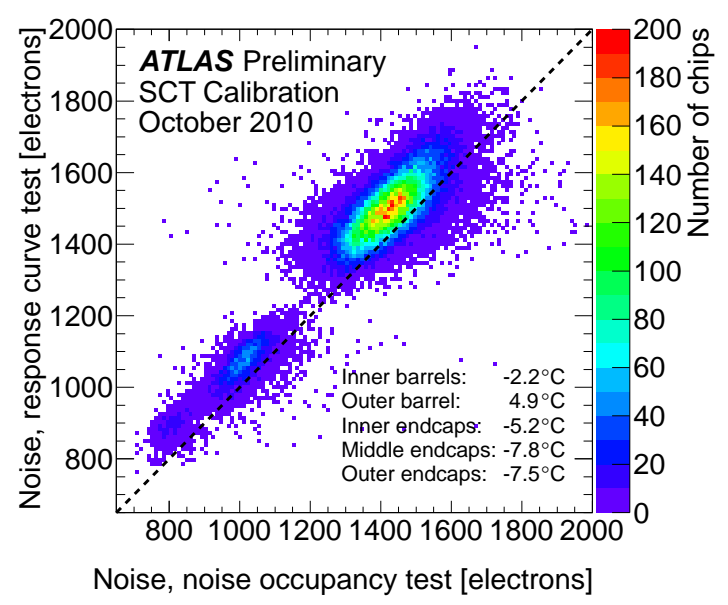

Figure 6: Comparison of noise measured with a charge injection circuit vs. noise occupancy in random triggers.

order of $10^{-5}$. The design specification [6] requires a noise occupancy of $<5 \cdot 10^{-4}$ indicated by the dotted line in Fig. 5. Only $0.2 \%$ of all strips of the SCT fail that criterium and consequently are disabled.

The two methods described above were compared several times with various running conditions and show good agreement (cf. Fig. 6).

\section{Hit Efficiency}

One important factor for the track reconstruction is the hit efficiency. It is defined as the number of hits on a track divided by the number of possible hits. In the latter, dead modules and chips are excluded. For example, the hit efficiency of the SCT for a run with $7 \mathrm{TeV}$ collisions taken in September 2010 is shown in Fig. 7. The efficiency is given for two different types of tracks: SCT standalone and Inner Detector combined tracks, both with the requirement of a transverse momentum of above $1 \mathrm{GeV}$. For standalone tracks at least $7 \mathrm{SCT}$ hits are demanded (not including the hit under test for efficiency) while for combined tracks at least 6 SCT Hits are required. The efficiencies in layer "0 inner" and "3 outer" are biased for the SCT standalone tracks as holes beyond the last measurement are not counted. The mean efficiency is around $99.9 \%$ for the barrel and $99.8 \%$ for the endcaps. All layers and disks are above $99.6 \%$ efficiency, exceeding the design specification of $99 \%$ [6]. The time stability of the mean efficiencies over the full 2010 dataset was about $\pm 0.1 \%$.

\section{Detector Operation}

Due to its very low noise level and high sensitivity the SCT is an excellent beam conditions monitor. This is especially important during LHC beam manipulating phases where other silicon precision detectors are switched off for safety. In contrast, the SCT can be run at a reduced voltage at which hit efficiencies of more than $90 \%$ are still achieved and noise levels are only slightly 

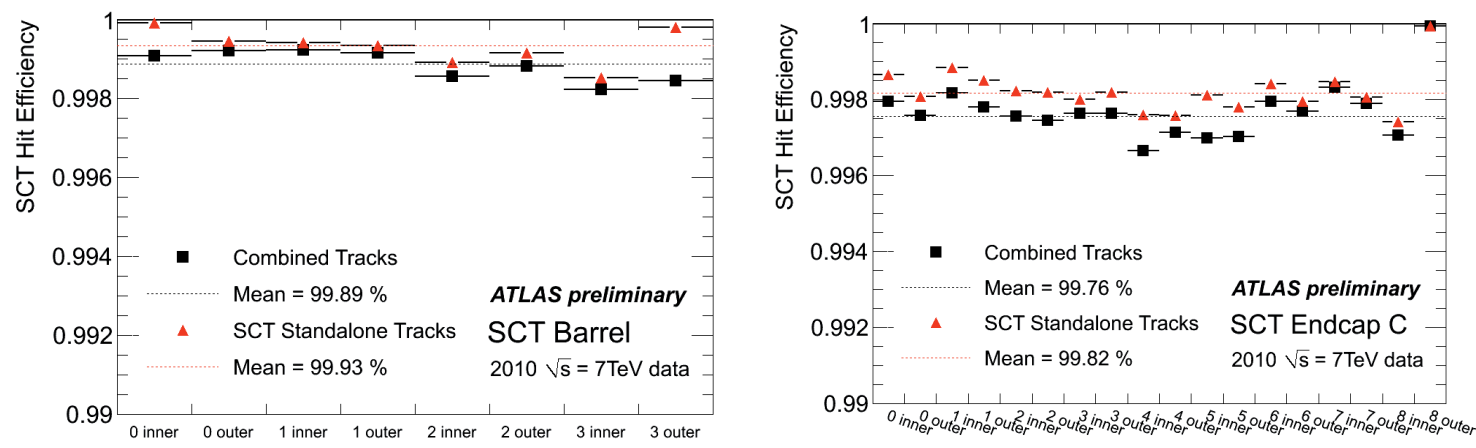

Figure 7: Hit efficiencies for the barrel layers (left) and endcap C disks (right). "Inner" corresponds to the side pointing to the vertex, "outer" is the side away from the vertex.

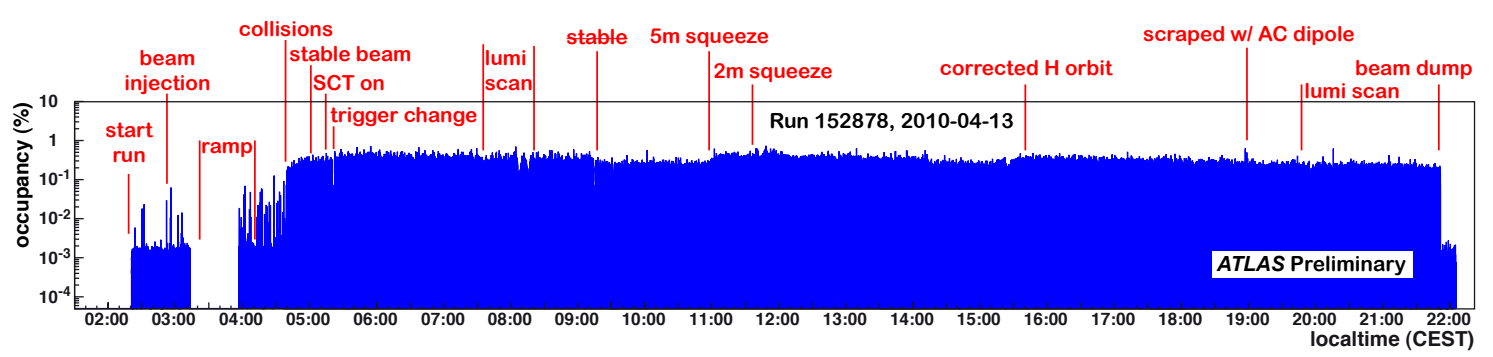

Figure 8: SCT occupancy measured in one ROS in a typical run in 2010. Major LHC changes are indicated.

increased (when considering coincidence hits on both module sides). In addition, the SCT is well timed-in (cf. Section 2) and has a very good time resolution. Hence, effects seen in the SCT occupancy can be directly correlated with beam manipulations or losses. As an example, Fig. 8 shows a long run with collisions. Cosmic showers before beam injection, beam-gas events after injection, and the start and stop of collisions can easily be identified. In contrast, all manipulations of the beam during collisions have very little effect on the occupancies (on a logarithmic scale). This indicates how stable and well-controlled the LHC beam is over a period of many hours.

After stable beams are declared by the LHC, the time the SCT (and Pixel detector) needs for turning on from standby to operating voltage is a critical factor for the ATLAS data taking efficiency. The usage of the SCT itself as a beam conditions monitor allows a safe and efficient warm start on the order of minutes.

Another important factor for operation is the SCT occupancy during collisions. Due to the increase in instantaneous luminosity and the thereby caused increased pile-up (i.e. several proton interactions per bunch crossing) the occupancies are steadily increasing since the start of the LHC. The event size limit in the ROSs is configured to $65 \mathrm{kB}$. This leads to event truncation, if the SCT reaches $46 \%$ occupancy. The average occupancy of the modules for events with 10 vertices (i.e. 10 pile-up collisions) was measured to be $0.7 \%$. The predicted mean occupancy at LHC design luminosity is about $1 \%$, thus far below the truncation limit. The largest occupancies observed in single events in proton-proton collisions were $20 \%$ for a single module and $8 \%$ on average over all 


\begin{tabular}{lrr}
\hline \hline Reason & Total Number & Percentage \\
\hline Cooling & 13 & $0.32 \%$ \\
Low Voltage & 7 & $0.17 \%$ \\
High Voltage & 6 & $0.15 \%$ \\
Readout & 4 & $0.10 \%$ \\
\hline Total & 30 & $0.73 \%$ \\
\hline \hline
\end{tabular}

Figure 9: Overview of reasons and numbers of disabled modules (Status: May 2010).

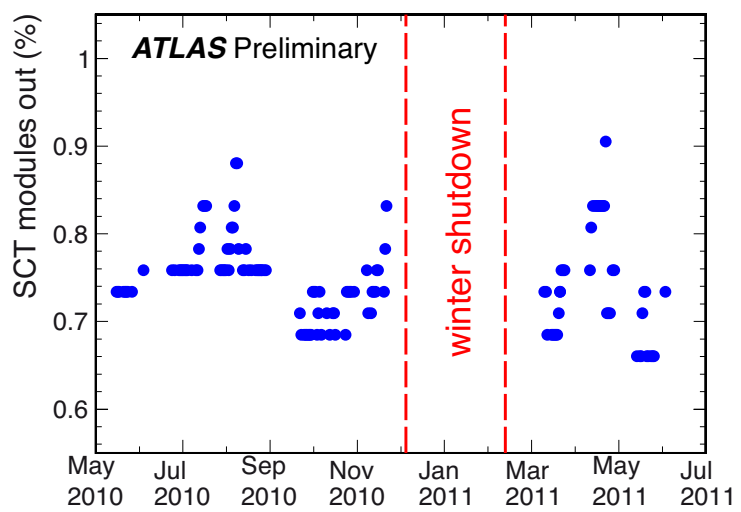

Figure 10: Percentage of disabled modules vs. time.

modules. Heavy ion collisions have in general a much denser particle track environment. In those collisions, SCT reaches occupancies of up to $37 \%$ in a single module and $16 \%$ on average over all modules. These values demonstrate that even extreme SCT occupancies do not limit the ATLAS data taking.

\section{Data Taking Stability}

The SCT is one of the most stable subdetectors in ATLAS. During the full 2010 run only 11 out of 196 runs had SCT issues. In 6 out of these 11 runs the data quality was unaffected. The other five runs had a BUSY signal issued by a ROD. Due to the geometry of the modules connected to a ROD, a BUSY affects an $\eta \phi$-region in the track reconstruction. If a ROD issues a BUSY signal, 48 modules are dropped from data taking. In 2010, a stopless removal procedure was put in place, so that this action could be performed on-the-fly without a need to stop the ATLAS run. However, a run start was needed to recover the ROD and its connected modules. An order of hours was needed for this recovery, depending on when a run start was possible. In 2011, a stopless recovery procedure was put into effect, allowing to reconfigure a ROD during an ongoing run. This reduced the recovery time to order of minutes.

On average in 2010 until mid-2011 $0.75 \%$ of modules were disabled during data taking and $0.18 \%$ had bytestream errors during a run. Reasons for disabling modules are given in Table 9. In the full 2010/2011 running, the SCT had a data taking efficiency of more than $99.4 \%$.

The most severe operational issue are TX deaths both in the Pixel and SCT detector. Both subdetectors use optical fibres to transfer data between the modules and the off-detector readout electronics. There is one TX fibre for clock and command and two RX fibres for data readout per module. In the SCT we have 360 off-detector TX arrays with 12 VCSELs per TX. In 2008, we were loosing the first time individual TX channels. Evidence pointed to ESD damage on VCSELs during manufacturing. ESD precautions were increased in the production of a new batch of TXs which was installed in summer 2009. Unfortunately, in May 2010, TXs started to fail again at a rate of order of 10 modules per week.

In contrast to the Pixel detector, the SCT has a redundancy scheme that largely reduces the effects of TX failures on data taking. If a TX dies, the commands can be send over the neighbouring 

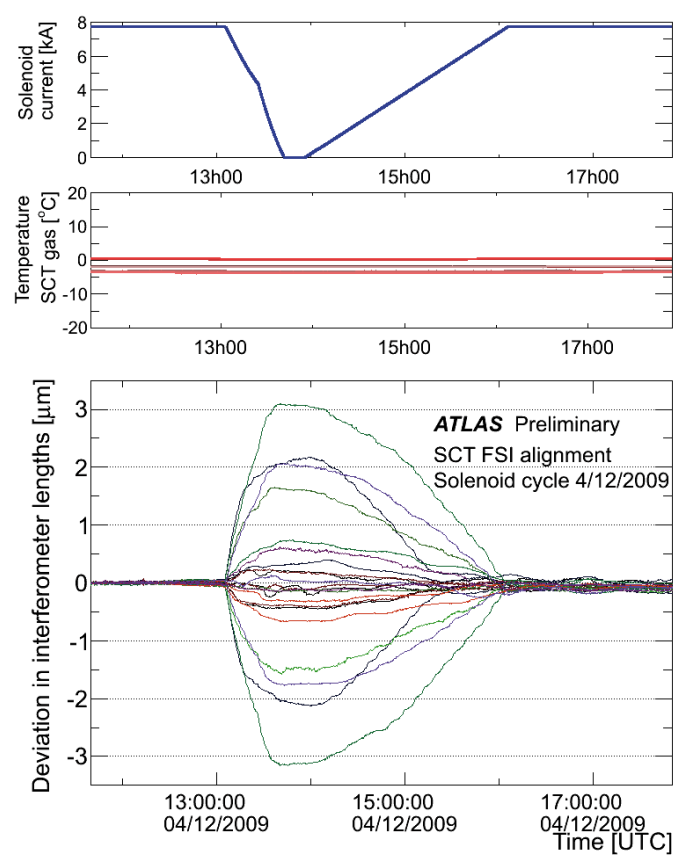

Figure 11: Position measurements of the SCT barrel flanges measured during a run including a full solenoid ramp cycle. Top: solenoid current, Middle: SCT gas temperature, Bottom: interferometer length deviations.

module and both modules stay fully operational. The configuration can be changed after a run stop, so that new modules affected by TX deaths are only missing from data taking in one run. If the neighbouring module already has a dead TX itself, or modules cannot use redundancy for setup reasons, the TX has to be replaced by a spare. This needs to wait up to order of days depending on when there is an opportunity for the change in the LHC schedule.

The cause of TX deaths was identified as ingress of humidity on the VCSELs. The current TXs seem to be not as hermetic as they should be. New packages are in preparation with increased humidity resistance and a lifetime of more than 10 years. As a precaution, currently dry air is used to flush the racks in order to reduce the relative humidity on the TXs.

Due to the redundancy scheme in the SCT data acquisition and the availability of spares, the number of disabled modules was kept below 1\% at all times in 2010-11 as can be seen in Fig. 10.

\section{Geometric Detector Stability}

To monitor the short- and long-term stability of the SCT geometry, an optical alignment system is installed in the detector. It is based on Frequency Scanning Interferometry [7] and consists of 842 interferometers that form a geodetic grid of distance measurements between various detector components. As an example, data of 24 out of 144 interferometers that monitor the relative position and orientation of the four carbon-fibre cylinders that support the barrel modules are included in Fig. 11. It depicts the movements of the SCT detector over a full cycle of the solenoidal field. As these can be caused both by temperature variations and changes in the magnetic field, the SCT temperature is also shown. It was constant over the time range of the plot. Before the ramp, the 
interferometers measure very stable detector positions with a standard deviation of about $11 \mathrm{~nm}$. In contrast, during the solenoid ramp they detect movements of up to $3 \mu \mathrm{m}$. Afterwards module positions return almost to their start values, where the standard deviation has increased to $49 \mathrm{~nm}$.

Continuous measurements since May 2009 have revealed that the SCT geometry is extremely stable with standard conditions. During non-stable periods that comprise cycles of the solenoidal and toroidal fields, cooling cycles, pixel calibration scans and the winter shutdown movements are typically less than $5 \mu \mathrm{m}$ between barrel layers.

\section{First Effects of Irradiation}

Radiation backgrounds induced by proton-proton collisions cause damage to the silicon sensors and electronics. Monitoring of their effects is needed to predict the future performance of the current and the upgraded SCT. Due to a linear relation between the leakage current and the radiation fluence, as long as the module temperature and depleted volume are constant, the on-detector fluence can be calculated by measuring the leakage current. In addition, radiation sensitive monitor packages (RadMons [8]) in the inner detector are constantly measuring the $1 \mathrm{MeV}$ neutron equivalent fluences directly. Figure 12, left shows a comparison between measured leakage currents in the barrel over time vs. predictions from a FLUKA simulation [9]. The temperature changes seen in the plot are taken properly into account. First of all, a rise in the leakage current due to the steep luminosity and thus radiation increase is clearly visible already at this early stage of irradiation. The agreement of prediction and measurement over time is excellent. Even effects caused by annealing are well simulated. The right-hand plot shows a geometrical map of the radiation flux. Ratios of measured divided by simulated fluences for individual barrel layers and endcap rings are given as well. The very good agreement for the four barrel layers is confirmed, ratios vary between 0.99 and 1.09. In the endcaps there is a good agreement between outer and middle rings (ratios 0.82-1.25). However, the inner rings located closest to the beampipe show larger deviations of up to a factor of two larger radiation in the measurement than in the simulation. This difference is currently under investigation. For the time being, the assumed safety factor of 1.5 in radiation for the development of the upgrade detector still seems to be sufficient. In June 2011, due to the increased leakage currents the trip limits had to be increased for the first time since the start of SCT operation.

\section{Conclusions}

The SCT is a performant, stably operating detector meeting all design specifications. Its properties and performance have been studied in different beam conditions. Its low noise level and high sensitivity have made it an important detector for beam conditions monitoring since the beginning of LHC commissioning. The number of disabled noisy strips is tiny (below $0.2 \%$ ) and all modules are well timed-in. The single hit efficiency of more than $99.6 \%$ in all layers and disks exceeds the design specification. The stopless removal and recovery of busy RODs is working without flaws and leads to a data taking efficiency of larger than $99.4 \%$. The cause of TX deaths was revealed to be humidity ingress in the VCSELs. The redundancy scheme has proven itself to be very helpful in this situation to keep the number of non-operating modules below $1 \%$ at all times. Enough spares are available to ensure a stable operation of the SCT detector and new TXs with improved humidity 

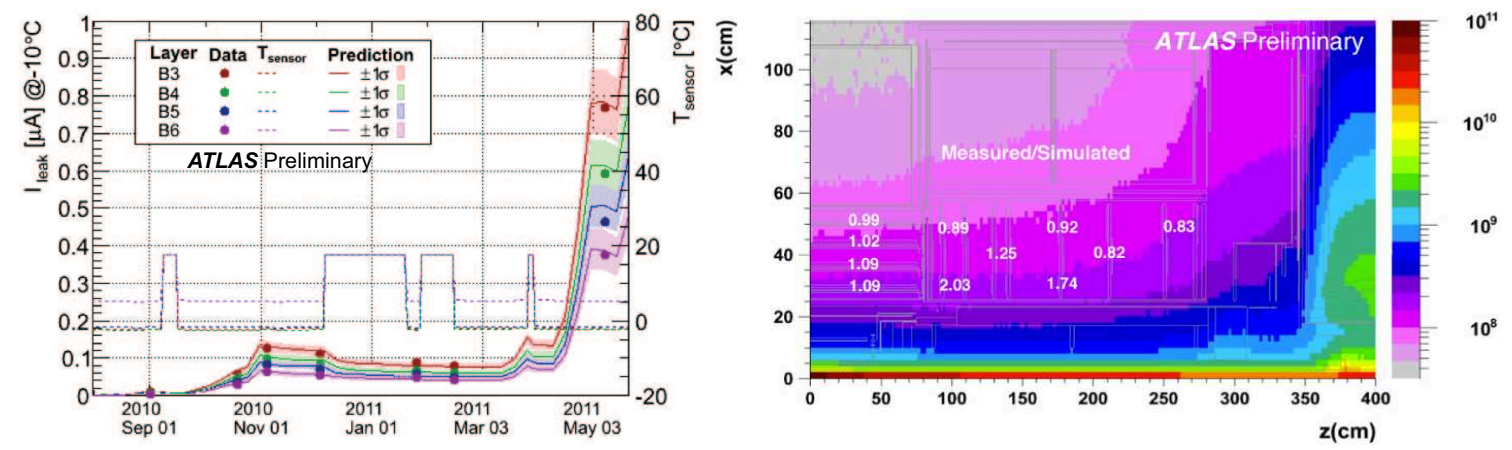

Figure 12: Left: Leakage current vs. time for the four barrel layers compared to the simulated FLUKA prediction [9]. The temperature of the modules is given in comparison. Right: Comparison of $1 \mathrm{MeV}$ neutron equivalent fluences determined from SCT leakage current measurements with predictions at $7 \mathrm{TeV}$. The colours indicate the fluences as determined by the FLUKA simulation. The values give the ratio of measured leakage current over FLUKA simulation for barrel layers and endcap disks. The beam axis is along the z-direction; zero corresponds to the interaction point.

resistance and thus increased lifetime are in production. The detector geometry is extremely stable during stable operating conditions. During non-standard conditions of magnetic fields and cooling movements of up to $5 \mu \mathrm{m}$ have been observed. First effects of irradiation have been observed in an increased leakage current, making higher trip limits necessary. The derived measurement of fluences agrees well with simulation within the safety factor of 50\% used for the upgrade development.

\section{References}

[1] The ATLAS Collaboration, The ATLAS experiment at the CERN Large Hadron Collider, JINST 3 (2008) S08003

[2] A. Abdesselam et al., The barrel modules of the ATLAS semiconductor tracker, Nucl. Instrum. Meth. A 568 (2006) 642

[3] A. Abdesselam et al., The ATLAS semiconductor tracker end-cap module, Nucl. Instrum. Meth. A 575 (2007) 352

[4] F. Campabadal et al., Design and performance of the ABCD3TA ASIC for readout silicon strip in the ATLAS semiconductor tracker, Nucl. Instrum. Meth. A 552 (2005) 561

[5] D. Attree et al., The evaporative cooling system for the ATLAS inner detector, JINST 3 (2008) P07003

[6] The ATLAS Collaboration, Inner Detector Techical Design Report, ATLAS TDR 4, CERN/LHCC/97-16, ISBN 92-9083-102-2

[7] S. Gibson et al., Coordinate measurement in 2-D and 3-D geometries using frequency scanning interferometry, Opt. Laser Eng. 44 (2005) 79-95.

[8] I. Mandic et al., Online integrating radiation monitoring system for the ATLAS detector at the Large Hadron Collider, IEEE Trans. Nucl. Sci. 54 (2007) 1143-1150.

[9] A. Fasso et al., FLUKA: a multi-particle transport code, CERN-2005-10 (2005), INFN/TC-05/11, SLAC-R-773 